\section{Promotion of Infection by Intravenously Administered Polysaccharides}

A VARIETY of polysaccharides shows infectionpromoting activity ${ }^{1-4}$. Nungester, Wolf and Jourdonais $^{1}$ noted that a crude gastric mucin preparation greatly lowered the minimum lethal dose of bacteria when the mucin together with the bacteria was given intraperitoneally, although it did not lower the minimum lethal dose sensibly when the mucin and the bacteria were given intravenously. This observation appears to be the basis of a belief that the infection-promoting activity is conditional upon the injection of the polysaccharide into a serous cavity ${ }^{5}$. The question whether a peritoneal infection can be promoted by a polysaccharide injected into the blood stream does not appear to have been examined critically. Since a number of polysaccharides are of interest as plasma volume expanders, this question might have clinical implications.

Infection-promoting potencies of dextran, levan, and mucin have been studied in mice given Salmonella typhi, 0.901, intraperitoneally. When mice (16-20 gm.) received intraperitoneally 0.1 minimum lethal dose of the bacteria, the mortality-rate was less than 5 per cent. When at the same time the mice also received $5 \mathrm{mgm}$. of native dextran or native levan, either intraperitoneally or intravenously, the infection was strongly promoted (mortality-rates greater than 70 per cent). Mucin, though similarly active when given intraperitoneally, was without activity when given intravenously. The peritoneum exudate was found to remain levan-free even after a massive dose (30 $\mathrm{mgm}$.) of native levan had been injected intravenously.

It is evident from these results that the promotion of infection by the neutral bacterial polysaccharides given intravenously does not require the local enclosure of the bacteria in the peritoneal space by an envelope of viscous polysaccharide. Whereas the neutral polysaccharides given intravenously appear to exert their influence on an extravascular in. fection, it need not be postulated that the hostcomponent inhibited by the polysaccharides is necessarily specifically extravascular. The levan failed to exert a decisive effect on intravenously inoculated bacteria. Hence it must fail to suppress at least one of the effective systemic anti-bacterial principles. As to dextran, Smith et al. ${ }^{3}$ have shown that it is without anti-complementary activity. We have found that, like mucin, native levan is lacking in infection-promoting activity when it is administered into the peritoneum of a mouse which has received an irritant (namely, a sub-lethal dose of bacteria) intraperitoneally 15 hours previously. This suggests that levan does not suppress the phagocytic activity of leucocytes which have gathered in the peritoneum in the course of the inflammatory process. In the light of these observations, it is perhaps permissible to speculate that the neutral polysaccharides, native dextran and native levan, are promoters of infection in the normal mouse because they modify the inflammatory process, with the result that transport of an anti-bacterial agent into the peritoneal space is restricted ${ }^{6-8}$.

Since infection-promoting activity is clearly undesirable in a clinical plasma-volume expander, it has been reassuring to find that acid hydrolysis leads to a rapid attenuation of the activity of both levan and dextran. Whereas the native levan and the native dextran with a molecular weight greater than $10^{6}$ were bath highly active as infection-promoters, the homologues with molecular weight in the range $10^{4}-10^{5}$ recovered from a partial acid hydrolysate of levan or dextran manifested a negligible infectionpromoting activity when given either intravenously or intraperitoneally.

The high potency of the neutral native bacterial polysaccharides (native levan, native dextran), their ready availability, adequate solubility, simple constitution, and homogeneous condition suggest that their use as promoters of infection in the place of the currently employed hog gastric mucin may offer worthwhile advantages. Since these neutral polysaccharides given intravenously are able to promote an extravascular infection, the latter is available in a form in which its responses to an antibiotic can be tested in the absence of an interfering viscous vehicle at the site of the infection. Furthermore, it may be anticipated that the range of action of the intravenously effective promoters will not be limited to peritoneally produced infections, but will also extend to infections produced at other vascularized sites.

\section{SнILO \\ D. FEINGOLD \\ SHLOMO HESTRIN}

Laboratory of Microbiological Chemistry of the Biochemistry Department,

Hebrew University-Hadassah Medical School, Jerusalem, Israel.

June 15.

Nungester, W., Wolf, A., and Jourdonais, L., Proc. Soc. Exp. Biol. and Med., 30,120 (1932).

Olitzki, L., Shelubsky, M., and Hestrin, S., Proc. Soc, Exp. Biol. and Med., 63, 491 (1916).

'Smith, H., Gallop, R., and Stanley, J., Biochem. J., 52, 15 (1952).

"Olitzki, L., Bact. Rev., 12, 149 (1948).

- Sandage, C., and Stark, O., J. Infect. Dis., 84, 310 (1949).

- Bennet, L., Proc. Soc. Exp. Biol. and Med., 81, 266 (1953).

7 Oerskov, J., Z. Immunitaetsforsch., 98, 359 (1940).

${ }^{6}$ Ercoli, N., Lewis, M., and Harker, E., Proc. Soc. Exp. Biol. and Med., 59, $273(1945)$.

\section{Heteropycnosis in the Autosome Segments of Ceriagrion coromandelianum (Odonata)}

THE sex-determining mechanism in dragon-flies consists, as a rule, of $X O-X X$ chromosomes, and the $X$-chromosome in the spermatocyte divides equationally in the first, and passes undivided to one pole or the other in the second, meiotic division ${ }^{1-4}$. In this respect, the $X$-chromosome of the male germcell in the Odonata behaves as in the Heteroptera, in which group also it usually divides in the first meiotic division only. We find that the chromosomes of the damsel-fly, Ceriagrion coromandelianum, exhibit another important feature in respect of which they resemble the hemipteran chromosomes; that feature is the presence of Feulgen-positive interconnecting threads joining the chromosomes at the spermatogonial metaphase, and also at the first and second meiotic metaphase. Such interconnecting chromatic threads are known to exist in many hemipterans ${ }^{5-8}$. Associated with the presence of these threads is a high degree of heteropycnosis of autosome segments. At pachytene the paired chromosomes carry deeply stained, condensed knobs. These heterochromatic segments occur in all chromosome pairs. The interconnecting threads, in our opinion, are derived from the chromosome matrix. It is well known that the protein framework of the heterochromatic chromosomes (or chromosome segments) differs from that of euchromatic chromosomes'. It is likely that 